\title{
Tweeting to Prepare: An Examination of Government and Organizational Messages during National Preparedness Month
}

\author{
Jenna L. Currie-Mueller \\ State University of New York at Oswego \\ Oswego, NY USA
}

\begin{abstract}
Each year, disasters have devastating consequences in the United States. Consequences are long term and extend beyond the disaster's immediate impact area. Establishing a culture of preparedness is necessary for the U.S. A prepared populace responds more effectively to disasters and is less stressful on community infrastructure and resources during the response phase. One of the ways government organizations and non-government organizations can encourage preparedness actions is via social media. This study examined preparedness messages existing independently of an emerging event disseminated on Twitter by government and non-government organizations. A total of 6,374 tweets were analyzed from data collected during National Preparedness Month. Tweets were analyzed for preparedness content and whether efficacy was included in preparedness messages.
\end{abstract}

Keywords - All-hazards, Disaster Communication, Efficacy, Preparedness, Social Media

SUGGESTED CITATION: Currie-Mueller, J. L. (2019). Tweeting to prepare: An examination of government and organizational messages during national preparedness month. Proceedings of the International Crisis and Risk Communication Conference, Volume 2 (pp. 15-17). Orlando FL: Nicholson School of Communication and Media. https://doi.org/10.30658/icrcc.2019.4

\section{INTRODUCTION}

Establishing a prepared populace is a proactive approach that is beneficial to the U.S. For example, a prepared populace reduces stress on community infrastructure during a disaster because people know what to do, how to do it, and have efficacy in doing so $[1,2]$. While preparedness messages can be transmitted by government and non-government organizations, responsibility lies within individuals and households $(\mathrm{I} / \mathrm{Hs})$ to engage in protective actions [3]. Unfortunately, I/Hs in the U.S. are underprepared [4], placing additional stress on response when a disaster occurs. Therefore, it is in the best interest for the U.S. to effectively communicate preparedness information to encourage its citizens to engage in protective action.

Social media may be helpful in promoting this endeavor. Research examining social media during a variety of events have identified social media's utility in communicating risk, crisis, and disaster messages. Regardless of platform, this research has birthed optimistic perspectives of social media's use outside the immediate pre-crisis and response phases. However, established literature has not examined preparedness messages that exist independently of an emerging event [5]. This research reports upon information disseminated by government and non-government accounts on Twitter during National Preparedness Month to determine the type of preparedness information communicated by organizational accounts and whether efficacy was incorporated into message content.

\section{NATIONAL PREPAREDNESS MONTH (NPM)}

NPM was launched in 2004 by the Federal Emergency Management Agency (FEMA). Multiple government agencies, official NPM coalition members, and other organizations participate each September in reminding Americans to prepare for events they are at risk for experiencing [6]. In 2017, NPM's official theme was "Disasters don't plan ahead. You can." Accompanying the theme was the campaign's official hashtag, \#PlanAhead, coordinating downloadable toolkits, and weekly themes focused on $\mathrm{I} / \mathrm{H}$ preparedness topics and community involvement [6]. 


\section{PREPAREDNESS}

Preparedness reflects a state of readiness to respond to an event to save lives and limit damage. It is an ongoing process incorporating planning, acquiring resources, and knowing what to do and when to do it [4]. Essentially, preparedness encourages being proactive in anticipating problems that occur during a disaster so that resources are allocated and in place during disaster response $[2,3]$. Overall. preparedness efforts at the $\mathrm{I} / \mathrm{H}$ level in the U.S. are lacking [3, 4]. When examined, preparedness is explored within a specific context (e.g. earthquakes, terrorism, etc.) or predictors for action are scrutinized. Unfortunately, examination of message content or delivery has not received as much attention.

\section{EFFICACY}

Individuals must believe they have the ability to undertake a recommended action, believe the action will work, or both. Messages that lack efficacy minimize I/H's risk perception; whereas, messages encouraging self-efficacy and response efficacy provide the ability for I/H's to make informed decisions [7]. Therefore, incorporating efficacy (self, response, or both) into risk messages is essential for organizations [8].

\section{METHODS}

Tweets using the hashtag, \#PlanAhead were collected during NPM from September 1, 2017 to September 30, 2017 using the web-based program TweetArchivist (www.tweetarchivist.com). This hashtag was selected because it was the official hashtag used and promoted by FEMA's Ready.gov NPM campaign. A total of 6,374 tweets from organizational accounts (federal government, local town/city government, county/regional government, state government, utilities, educationrelated, for-profit, non-profit, consulting, media, military, or other) were used for analysis.

Tweets were coded for efficacy and preparedness content by two independent coders. Tweets including information that could encourage self- or response efficacy were categorized as communicating efficacy [8]. Tweets were coded as either containing or not containing efficacious information. Coder agreement in this category reflected excellent agreement beyond chance for reliability $(k=.94)$ and $\operatorname{drift}(k=.88)$ checks.

Tweets coded for preparedness content identified content as communicating general preparedness information, specifichazard information, or N/A. General preparedness tweets reflected an all-hazards approach to communicating preparedness, specific-hazard category identified a specific hazard, and N/A communicated information not reflective of the aforementioned categories. Coder agreement for preparedness content was high in reliability $(k=.90)$ and drift $(k=.82)$.

\section{RESULTS}

A large proportion of the dataset contained preparedness information ( $n=5560,87.7 \%)$. Significant differences in proportions were evident, $x^{2}(2, N=6342)=4919.36, \mathrm{p}<.001$ with general preparedness information accounting for $74.9 \%(n=4747)$, specific-hazard preparedness information accounting for $12.8 \%(n=813)$, and non-preparedness messages accounting for $12.3 \%(n=782)$ of tweets disseminated by government and non-government organizational accounts.

Efficacy was included in a large proportion of the analyzed tweets $(n=4770,85.7 \%)$ compared to tweets not incorporating efficacy $(n=790,14.3 \%), x^{2}(1, n=5560)=2851.86, p<.01$.

\section{DISSCUSSION}

This research examined preparedness messages disseminated on Twitter by government and non-government organizations during NPM. In examining message dissemination during this timeframe, the contribution of this research is twofold: it provides insight into the content of preparedness messages on social media not associated with a specific and emerging event and it extends understanding of efficacy and preparedness messages.

Social media provides a platform for government and non-government organizations to communicate about preparedness information during a non-specific event. In cultivating a culture of preparedness in the U.S., disseminating preparedness messages outside of a specific event (like a hurricane) is essential because $\mathrm{I} / \mathrm{Hs}$ are able to have meaningful access to information that communicates what to do, how to do it, and when to perform the action. Whereas this type of information may not be available to I/Hs during an imminent event due to the volume of messages being transmitted.

In regards to volume, general preparedness messages were the most commonly disseminated messages in the data. This reflects alignment with the U.S.' all-hazards approach to emergency management. During a crisis, scholarship strongly suggests an organization to maintain control of the narrative. Within this research, the narrative of all-hazards was dominant across government and non-government organizations, suggesting FEMA to have maintained control of the primary approach to communicating about preparedness information.

Overall, the participation of multiple government and non-government organizations demonstrates execution of Lin et al's [9] best practices of social media, owning the hashtag. Previous research not specific to preparedness has revealed lack of participation in social media by government agencies - even when they were the ones to promote an official hashtag [10]. Yet, when considering the data collected for this research was every hour throughout NPM, the total volume of tweets 
sent by government and non-government accounts suggests perhaps the best practice of being cautious about message speed [9] may not translate fully to non-event preparedness in that the data suggests organizations may have been a little too cautious.

Since preparedness is an ongoing process, it would be prudent for organizations to communicate preparedness information outside of NPM. NPM is a great opportunity for organizations to gain I/Hs' attention and organizations may be able to use this event to provide a foundation to continue the conversation year round.

Efficacy was included in both general preparedness messages and specific-hazard messages. While efficacy is necessary in communicating about risk [7, 8], it often is not present in messages. For example, in Vos and Buckner's [8] examination of the emergence of bird flu messages on Twitter, efficacy was present in only a few messages. However, specific to this research, the data suggests efficacy to have been significantly present in messages disseminated by government and nongovernment organizations. This may be due to the general nature of preparedness and campaigning for $\mathrm{I} / \mathrm{Hs}$ to undertake proactive protective actions. Overall, the general theme for NPM reflected efficacy in its tagline by encouraging individuals to plan ahead. This may have contributed to the high frequency of messages that incorporated efficacy and efficacy should be examined against all stages of the disaster life cycle.

Regardless of impetus for efficacy being incorporated into preparedness messages, efficacy is necessary to include in messages that recommend a particular action [7]. This is especially true when trying to engender a cultural shift from reactive to proactive measures. Including efficacy, (self-, response, or both) is beneficial in promoting protective actions.

\section{CONCLUSION}

It is necessary for the U.S. to establish a culture of preparedness. Communicating via social media platforms, like Twitter, provide government and non-government organizations the opportunity to disseminate preparedness messages to a large audience during times of a non-event. Specific to this research, the data suggested general preparedness messages to have been significantly disseminated more than specific-hazard preparedness messages. This reflects alignment with the allhazards approach to disaster management adopted by the U.S. Further, efficacy was evident in a large proportion of both general and specific-hazard tweets in the dataset. This suggests preparedness messages communicated by government and non-government organizations encouraged I/Hs to take recommended actions included in the preparedness message.

\section{Author Biography}

Jenna L. Currie-Mueller (mjenna.curriemueller@oswego.edu, M.S., North Dakota State University, 2014) is an Assistant Professor in the Department of Communication Studies at the State University of New York at Oswego in Oswego, NY.

\section{REFERENCES}

[1] O’Brien, G., O'Keefe, Gadema, Z., \& Swords, J. (2010). Approaching disaster management through social learning. Disaster Prevention and Management: An International Journal, 19, 498-508. https://doi.org/10.1108/09653561011070402

[2] Quarantelli, E. L. (2000). Another selective look at future social crisis: Some aspects of which we can already see in the present. (Preliminary paper \#298). Disaster Research Center (DRC). University of Delaware.

[3] Tierney, K. J., Lindell, M. K., \& Perry, R. W. (2001). Facing the unexpected: Disaster preparedness and response in the United States. Washington, D.C.: Joseph Henry Press.

[4] Donahue, A. K., Eckel, C. C., \& Wilson, R. K. (2014). Ready or not? How citizens and public officials perceive risk and preparedness. American Review of Public Administration, 44(4S), 89S-111S. https://doi.org/10.1177/0275074013506517

[5] Alexander, D. E. (2014). Social media in disaster risk reduction and crisis management. Science and Engineering Ethics, 20(3), 717-733. https://doi.org/10.1007/s11948-013-9502-z

[6] Ready. (September, 2017). National preparedness month. Retrieved from http://www.ready.gov/september

[7] Sellnow, T. L., Ulmer, R. R., Seeger, M. W., \& Littlefield, R. S. (2009). Effective risk communication: A messagecentered approach. New York, NY: Springer.

[8] Vos, S. C., \& Buckner, M. M. (2016). Social media messages in an emerging health crisis: Tweeting bird flu. Journal of Health Communication, 21, 301-308. https://doi.org/10.1080/10810730.2015.1064495

[9] Lin, X., Spence, P. R., Sellnow, T. L., \& Lachlan, K. A. (2016). Crisis communication, learning and responding: Best practices in social media. Computers in Human Behavior, 65, 601-605. https://doi.org/10.1016/j.chb.2016.05.080

[10] Lachlan, K. A., Spence, P. R., Lin, X., Najarian, K., \& Del Greco, M. (2014). Twitter use during a weather event: Comparing content associated with localized and non-localized hashtags. Communication Studies, 65, 519-534. https://doi.org/10.1080/10510974.2014.956940 